\title{
Das Navi
}

Liebe Leserinnen, liebe Leser,

man macht sich seine Sorgen. Wie man weiß, hat auch die moderne Kommunikationstechnik nicht nur ihr Gutes sondern auch ihre Schattenseiten. Die Mobiltelephonie z.B.: Man kann sich mit ihr von überall her mitteilen, ist überall erreichbar - was ja überwiegend gut ist - aber man kann auch als Träger eines Handys geortet und so per Funk verfolgt werden. Man kann anonym belästigt und getäuscht werden etc. Oder: Was kann nicht alles dem Internet-Teilnehmer zustoßen! Der vorsichtige Mensch traut also diesen neuen Diensten nicht, solange er nicht auch ihre Kehrseite betrachtet hat.

Ich möchte hier auf die satellitengestützte Navigation bzw. das Navi (Navigationsgerät) eingehen. Das ist bei Autofahrern sehr beliebt. Man kauft sich eines, gibt ihm die Urlaubsadresse ein, macht es an der Windschutzscheibe fest und lässt sich per bewegter graphischer Anzeige und ansprechender Frauenstimme korrekt und höflich bis an das bezeichnete Haus führen. Das macht das Reisen angenehm. Es kann zwar auch Einbrechern zugute kommen: ein Nachteil, der hier weiter keine Rolle spielen soll. Nein, hier geht es um Sicherheitsbedenken, die man bezüglich der Satelliten haben könnte, gibt es doch anderorts Satelliten, die zur Ausforschung der Erdoberfläche eingesetzt werden. Könnte es nicht sein, dass ein Satellit den Autofahrer lenkt, dabei zwangsläufig seinen Weg und die Umstände erfasst und seine personenbezogenen Daten weitergibt?

Dazu kurz zum GPS General Positioning System: Es erfordert 24 Satelliten, die etwa $20.000 \mathrm{~km}$ hoch, jeweils zu viert gleich verteilt in einer von insgesamt sechs Umlaufbahnen die Erde umkreisen. Die Bahnebenen schneiden in gleichem Abstand zueinander die Äquatorebene in einem Winkel von $55^{\circ}$. Ein Umlauf dauert etwa einen Tag. Jeder Satellit sendet Signale aus, denen seine Bahndaten und deren Sendezeitpunkt zu entnehmen sind. Diese Signale werden vom Navi empfangen. Aus der Differenz des Empfangszeitpunkts zum Sendezeitpunkt und aus der Lichtgeschwindigkeit berechnet das Navi die Entfernung zum Satelliten. Aus den Entfernungen zu drei verschiedenen Satelliten und deren Bahndaten kann es trigonometrisch seine geographische Position bestimmen, diese in sein Kartenwerk projizieren und dort den Ort des Kraftfahrzeugs erkennen. Ein viertes Satellitensignal ist erforderlich, um die Uhren zu synchronisieren und Verringerungen der Lichtgeschwindigkeit in der Iono- und er Troposphäre auszugleichen. Mit dieser zusätzlichen vierten Messung lässt sich auch die Höhe des Navigationsgeräts über dem Meeresspiegel errechnen. Aus anderen Signaleigenschaften (Dopplerverschiebung) kann das Gerät auch seine Fahrgeschwindigkeit bestimmen.

Alles, was das GPS-Satellitensystem zur Navigation beiträgt, sind die fortlaufenden Signale der vier Satelliten. Alles andere - Routenberechnung, Benutzeroberfläche und die unterschiedlichen Dienstleistungen - ist Sache des Navis. Es empfängt die Signale der Satelliten, sendet aber an sie keinerlei Signale zurück, auch nicht an irgendeine andere Stelle. Es teilt sich mit seinen Fahranweisungen ausschließlich seinem Anwender mit. Die Satelliten dienen inm lediglich zur Orientierung, wie etwa dem Wanderer Sonnenstand und Uhr. Das GPS-System ist in diesem Sinne ein Rundfunksystem, das nicht nur das Navi sondern jeden bedient, der seine Signale empfangen und auswerten kann. Seine Nutznießer - Navi, Fahrzeug, Fahrer - beachtet es nicht. Sie sind ihm unbekannt; entsprechend auch ihre Position, Geschwindigkeit und Route.

Man braucht also, liebe Leserinnen und Leser, nicht zu befürchten, dass mit dem Einschalten des Navigationsgeräts irgendwelche Daten nach außen gelangen; insbesondere auch keine personenbezogenen. Hier liegt also eine Kommunikationstechnik vor, die erfreulicherweise für den Datenschutz keine Schattenseiten bietet.

Mit freundlichen Grüßen, Ihr

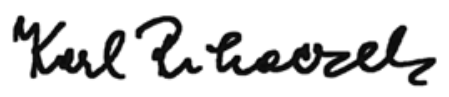

